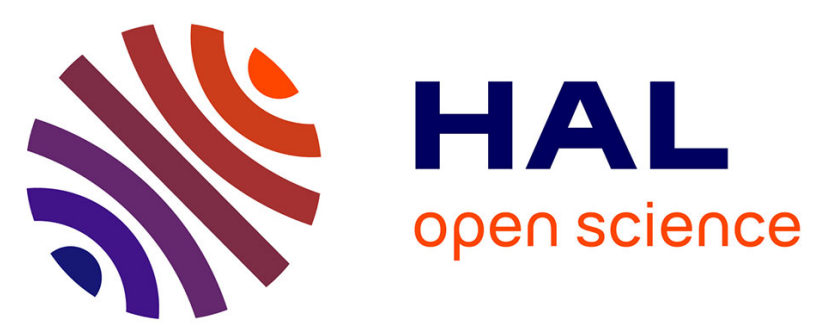

\title{
Azobenzene as Light-Activable Carrier Density Switches in Nanocrystals
}

Bertille Martinez, Rémi Plamont, Charlie Gréboval, Prachi Rastogi, Yoann

Prado, Junling Qu, Audrey Chu, Clément Livache, Xiangzhen Xu, Hervé

Cruguel, et al.

\section{To cite this version:}

Bertille Martinez, Rémi Plamont, Charlie Gréboval, Prachi Rastogi, Yoann Prado, et al.. Azobenzene as Light-Activable Carrier Density Switches in Nanocrystals. Journal of Physical Chemistry C, 2019, 10.1021/acs.jpcc.9b08597 . hal-02314917

\section{HAL Id: hal-02314917 \\ https://hal.science/hal-02314917}

Submitted on 10 Jul 2020

HAL is a multi-disciplinary open access archive for the deposit and dissemination of scientific research documents, whether they are published or not. The documents may come from teaching and research institutions in France or abroad, or from public or private research centers.
L'archive ouverte pluridisciplinaire HAL, est destinée au dépôt et à la diffusion de documents scientifiques de niveau recherche, publiés ou non, émanant des établissements d'enseignement et de recherche français ou étrangers, des laboratoires publics ou privés. 


\title{
Azobenzene as Light-Activable Carrier Density Switches in Nanocrystals
}

Bertille Martinez ${ }^{1,2}$, Rémi Plamont ${ }^{3}$, Charlie Greboval ${ }^{1}$, Prachi Rastogi ${ }^{1}$, Yoann Prado ${ }^{1}$, Junling Qu $^{1}$, Audrey $\mathrm{Chu}^{1}{ }^{1}$, Clément Livache ${ }^{1,2}$, Xiang Zhen $\mathrm{Xu}^{2}$, Hervé Cruguel ${ }^{1}$, Sandrine Ithurria ${ }^{2}$, Mathieu G. Silly ${ }^{5}$, Nicolas Goubet ${ }^{4}$, Emmanuel Lhuillier ${ }^{1 *}$

${ }^{1}$ Sorbonne Université, CNRS, Institut des NanoSciences de Paris, INSP, F-75005 Paris, France.

${ }^{2}$ Laboratoire de Physique et d'Etude des Matériaux, ESPCI-Paris, PSL Research University, Sorbonne Université Univ Paris 06, CNRS UMR 8213, 10 rue Vauquelin 75005 Paris, France.

${ }^{3}$ Bio-inspired and Smart Materials, MESA+ Institute for Nanotechnology, University of Twente, PO Box 217, 7500 AE Enschede, The Netherlands

${ }^{4}$ Sorbonne Université, CNRS, De la Molécule aux Nano-objets: Réactivité, Interactions et Spectroscopies, MONARIS, F-75005 Paris, France.

${ }^{5}$ Synchrotron-SOLEIL, Saint-Aubin, BP48, F91192 Gif sur Yvette Cedex, France.

\begin{abstract}
Control of carrier density in colloidal quantum dots is a major challenge for their integration into optoelectronic devices. Several chemical methods have been proposed to reach this goal including: introduction of impurities, non-stoichiometric compounds, introduction of redox molecules as ligands and surface gating obtained by tuning the dipole associated with surface ligands. None of these techniques allows post synthesis tunability. Alternatively, optical pumping requires high excitation power which may heat and finally damage the sample. Here, we propose a new procedure based on grafting of azobenzenes (AZBs) on the nanocrystal surface. The AZBs have two conformations (cis and trans), which are associated with strongly different dipole moments. The transition from one conformation to the other can be activated using UV or visible light at low intensities $\left(<100 \mathrm{~mW} \cdot \mathrm{cm}^{-2}\right)$. Grafting the AZBs on the nanocrystal surface leads to a light-tunable surface dipole, which shifts the nanocrystal bands and lead to a tunable carrier density. We apply this method to p-type HgTe and degenerately n-doped HgSe nanocrystals. We demonstrate, thanks to transport measurements, a change of the carrier density corresponding to a band shift up to $40 \mathrm{meV}$.
\end{abstract}

To whom correspondence should be sent: el@insp.upmc.fr 


\section{INTRODUCTION}

Nanocrystals (NCs) appear as an important building block for solution-processed optoelectronic devices ${ }^{1,2}$ such as light emitting diodes, ${ }^{3}$ solar cells ${ }^{4}$ and infrared (IR) sensors. ${ }^{5,6}$ In this quest, the doping control becomes of utmost interest: it is critical for the design of $p-n$ junctions, ${ }^{7}$ to reduce lasing threshold ${ }^{8}$ or to induce intraband absorption in the mid infrared. ${ }^{9,10}$ Doping of NCs can be obtained from various methods: $:^{11-13}$ introduction of extrinsic impurities ${ }^{14-16}$ within the NCs or within the array of nanocrystals, non-stoichiometry of the material $\left(\mathrm{Cu}_{2-\mathrm{x}} \mathrm{S}_{1}{ }^{17}(\mathrm{Bi} ; \mathrm{Sb})_{2} \mathrm{Te}_{3}, \mathrm{HgSe}^{18,19}\right)$, metal functionalization, ${ }^{20,21}$ functionalization by redox molecules ${ }^{22,23}$ or surface dipole functionalization. ${ }^{24-28}$ In this latter case, the dipole moment associated with the grafted ligand on the nanocrystal surface induces a band shift $(\Delta \mathrm{E})$ according to the following equation $\Delta \mathrm{E}=\frac{\sigma \mu}{\varepsilon_{0} \varepsilon_{r}}$ with $\sigma, \mu, \varepsilon_{0}$ and $\varepsilon_{r}$ being respectively the dipole surface density, the dipole magnitude, the vacuum permittivity and the dielectric constant.

Once under thin film form, all these techniques suffer from a lack of tunability. Alternatively, tuning carrier density can be achieved thanks to gating in a field effect transistor ${ }^{29,30}$ configuration or with an electrochemical cell. ${ }^{10}$ Optical pumping can also be used to fill the conduction band and so saturate the interband absorption. In this case the carrier density tuning is either short-lived when pulsed excitation is used, or requires high power continuous wave (CW) excitation, which may damage the nanocrystal array due to sample heating.

Here, we explore an alternative approach for the control of carrier density with light, which is reversible and compatible with low excitation power $\left(<100 \mathrm{~mW} \cdot \mathrm{cm}^{-2}\right.$ ). To do so, we functionalize NCs with azobenzenes (AZBs), see Figure $1 .{ }^{31,32}$ The reversible nitrogen-nitrogen double-bond configuration can switch from "cis" to "trans" and is triggered respectively by UV (cis to trans) and visible (trans to cis) irradiations, which makes AZBs promising molecular switches or tools to induce self-assembly by external stimuli. ${ }^{33}$ During conformation change, the dipole associated to the molecule is dramatically affected and changes by $\approx 3$ Debye,$^{34}$ see Figure $2 \mathrm{c}$ and e. Thus, by using AZBs as a surfacecapping molecules for nanocrystals, we propose a method where the nanocrystals surface dipole is light reconfigurable. Compared to conventional optical excitation, this method can be operated under much lower excitation power and so reduces the material degradation. We bring evidence that it is possible to control the response of infrared nanomaterials while shining light (with low-power excitation) in the UV.

\section{METHODS}

\section{Synthesis of thiol functionalized azobenzenes}

Materials for thiol functionalized azobenzenes: All reactions are carried under $\mathrm{N}_{2}$. THF was purified and dried over Braun solvent purification system (MB-SPS-800), other solvents $\left(\mathrm{CH}_{2} \mathrm{Cl}_{2}\right.$, heptane, methanol, ethyl acetate) were purchased dry from Sigma-Aldrich and used without further purification. The chemical reagents were purchased from Synthon, abcr, Sigma-Aldrich and used without further purifications.
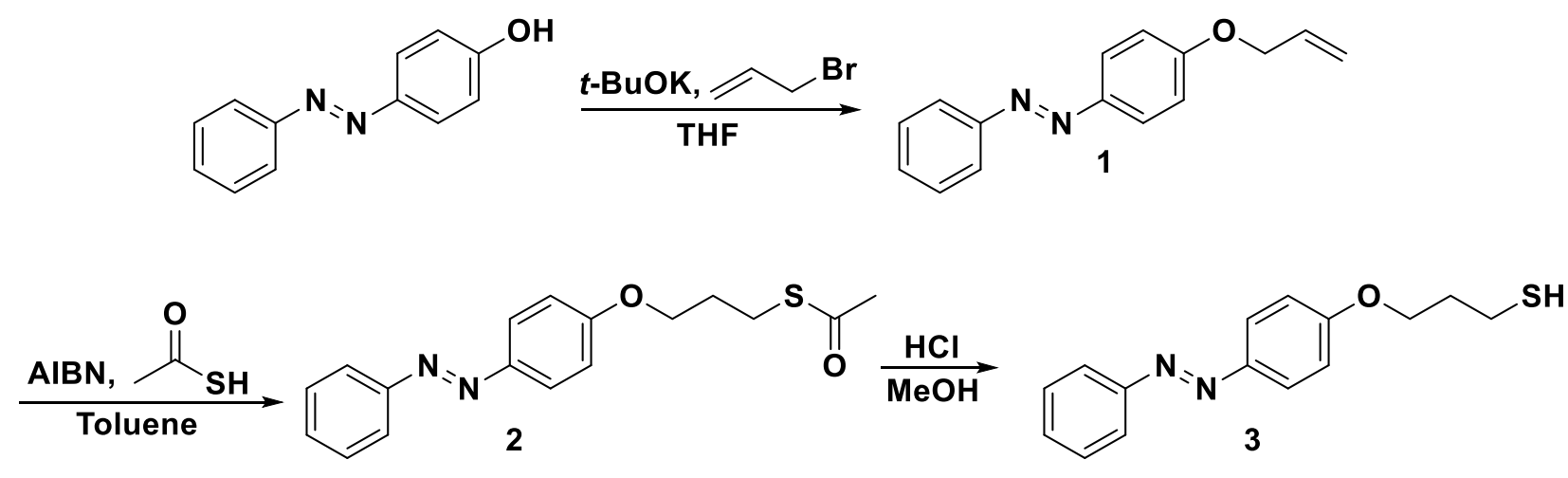

Figure 1: Synthetic route to thiol functionalized azobenzene. 
Compound 1: 4-(allyloxy)phenyl-2-phenyldiazene: In a dried three neck round bottom flask, 4-(phenyldiazenyl)phenol (1.58 g, $8 \mathrm{mmol}, 1$ eq.), 3-bromoprop-1ene $(0.7 \mathrm{~mL}, 0.97 \mathrm{~g}, 8 \mathrm{mmol}, 1$ eq.) and potassium t-butoxide (1 g, $10.4 \mathrm{mmol}$, 1.3 eq.) were dissolved in $20 \mathrm{~mL}$ of dry THF. The reaction mixture was stirred at reflux for 16 hours after what it was cool down to room temperature. The solvent was evaporated under reduced pressure and the residue dissolved in $100 \mathrm{~mL} \mathrm{CH}_{2} \mathrm{Cl}_{2}$. The organic phase was washed with distilled water $(3 \times 60 \mathrm{~mL})$. The organic phase was dried over $\mathrm{MgSO}_{4}$ and the solvent was evaporated under reduced pressure. The product was purified by flash chromatography on silica with ethyl acetate / heptane (1:9) as eluent. The product was collected as orange solid, $1.525 \mathrm{~g}$, yield $80 \%$.

${ }^{1} \mathrm{H} \mathrm{NMR}\left(400 \mathrm{MHz}, \mathrm{CDCl}_{3}\right) \delta=4.62\left(\mathrm{t}, 2 \mathrm{H}, \mathrm{O}-\mathrm{CH}_{2}\right), 5.39\left(\mathrm{~m}, 2 \mathrm{H},=\mathrm{CH}_{2}\right), 6.08(\mathrm{~m}, 1 \mathrm{H},=\mathrm{CH}-), 7.04(\mathrm{~d}, 2 \mathrm{H}, \mathrm{Ar}-\mathrm{H}), 7.50(\mathrm{~m}$, $3 \mathrm{H}, \mathrm{Ar}-\mathrm{H}), 7.91 \mathrm{ppm}(\mathrm{m}, 4 \mathrm{H}, \mathrm{Ar}-\mathrm{H})$.

Compound 2: 3-(4-phenyldiazenyl)phenoxy)propyl-ethanethioate: In a dried three neck round bottom flask, compound 1 ( $1 \mathrm{~g}, 4.2 \mathrm{mmol}, 1$ eq.) and AIBN (1.45 g, $8.8 \mathrm{mmol}, 2.1$ eq.) were dissolved in $20 \mathrm{~mL}$ of dry toluene. Thioacetic acid $(0.63 \mathrm{~mL}, 0.67 \mathrm{~g}, 8.8 \mathrm{mmol}, 2.1 \mathrm{eq}$.) was added dropwise to the mixture under stirring. The reaction mixture was stirred at reflux for 16 hours. The reaction was then cool down to room temperature and diluted with 60 $\mathrm{mL}$ of toluene. The organic phase was washed with saturated $\mathrm{NaHCO}_{3}$ solution in water $(60 \mathrm{~mL})$ and distilled water $(2$ $x 60 \mathrm{~mL}$ ). The organic phase was dried over $\mathrm{MgSO}_{4}$ and the solvent was evaporated under reduced pressure. The product was purified by flash chromatography on silica with ethyl acetate / heptane (1:9) as eluent. The product was collected as orange solid, $1 \mathrm{~g}$, yield $76 \%$.

${ }^{1} \mathrm{H}$ NMR $\left(400 \mathrm{MHz}, \mathrm{CDCl}_{3}\right) \delta=2.12\left(\mathrm{~m}, 2 \mathrm{H}, \mathrm{C}-\mathrm{CH}_{2}-\mathrm{C}\right), 2.36\left(\mathrm{~s}, 3 \mathrm{H},-\mathrm{COCH}_{3}\right), 3.09\left(\mathrm{t}, 2 \mathrm{H}, \mathrm{CH}_{2}-\mathrm{S}\right), 4.10\left(\mathrm{t}, 2 \mathrm{H}, \mathrm{O}-\mathrm{CH}_{2}\right), 7.01$ (d, $2 \mathrm{H}, \mathrm{Ar}-\mathrm{H}), 7.50(\mathrm{~m}, 3 \mathrm{H}, \mathrm{Ar}-\mathrm{H}), 7.88 \mathrm{ppm}(\mathrm{m}, 4 \mathrm{H}, \mathrm{Ar}-\mathrm{H})$.

Compound 3: 3-(4-(phenyldiazenyl)phenoxy)propane-1-thiol: Compound 2 (0.5g, 1.6 mmol, 1 eq.) was dissolved in $40 \mathrm{~mL}$ of degassed $\mathrm{MeOH}$ under $\mathrm{N}_{2} .2 \mathrm{~mL}$ of $\mathrm{HCl}$ (37\%) (14 eq.) were added dropwise and the reaction mixture was refluxed for $3 \mathrm{~h}$. The reaction was then cool down to room temperature and the excess $\mathrm{HCl}$ was neutralized with a $\mathrm{NaOH}$ solution in water (1M). $100 \mathrm{~mL}$ of $\mathrm{CH}_{2} \mathrm{Cl}_{2}$ were added to the reaction mixture and the organic phase was washed with distilled water $(2 \times 100 \mathrm{~mL})$. The organic phase was collected and dried over $\mathrm{MgSO}_{4}$ and the solvent was evaporated under reduced pressure. The product was purified by flash chromatography on silica with ethyl acetate / heptane (1:15) as eluent. The product was collected as orange solid, $0.260 \mathrm{~g}$, yield $60 \%$.

${ }^{1} \mathrm{H} \mathrm{NMR}\left(400 \mathrm{MHz}, \mathrm{CDCl}_{3}\right) \delta=1.42(\mathrm{t}, 1 \mathrm{H},-\mathrm{SH}), 2.12\left(\mathrm{~m}, 2 \mathrm{H}, \mathrm{C}-\mathrm{CH}_{2}-\mathrm{C}\right), 2.76\left(\mathrm{~m}, 2 \mathrm{H}, \mathrm{S}-\mathrm{CH}_{2}\right), 4.16\left(\mathrm{t}, 2 \mathrm{H}, \mathrm{O}-\mathrm{CH}_{2}\right), 7.02(\mathrm{~d}$, $2 \mathrm{H}, \mathrm{Ar}-\mathrm{H}), 7.50(\mathrm{~m}, 3 \mathrm{H}, \mathrm{Ar}-\mathrm{H}), 7.92 \mathrm{ppm}(\mathrm{m}, 4 \mathrm{H}, \mathrm{Ar}-\mathrm{H})$, see Figure $\mathrm{S} 4$.

${ }^{13} \mathrm{C} \mathrm{NMR}\left(100 \mathrm{MHz}, \mathrm{CDCl}_{3}\right) \delta=21.30\left(\mathrm{CH}_{2}-\mathrm{S}\right), 33.34\left(\mathrm{C}_{-} \mathrm{CH}_{2}-\mathrm{C}\right), 66.06\left(\mathrm{CH}_{2}-\mathrm{O}\right), 114.81\left(\mathrm{C}_{\mathrm{Ar}}\right), 122.68\left(\mathrm{C}_{\mathrm{Ar}}\right), 124.87\left(\mathrm{C}_{\mathrm{Ar}}\right)$, $129.14\left(C_{A r}\right), 130.49\left(C_{A r}\right), 147.15\left(C_{A r}-N\right), 152.85\left(C_{A r}-N\right), 161.41 \mathrm{ppm}\left(C_{A r}-O\right)$, see Figure $S 5$.

\section{Nanocrystal syntheses}

Chemicals: For nanocrystals, the following list of product have been used: mercury acetate $\left(\mathrm{Hg}(\mathrm{OAc})_{2}\right.$, Sigma-Aldrich), mercury chloride $\left(\mathrm{HgCl}_{2}\right.$, Sigma-Aldrich, 99\%), tellurium powder ( $\mathrm{Te}$, Sigma-Aldrich, 99.99\%), selenium powder (Se, Strem Chemicals), oleic acid (OA, Sigma-Aldrich, 90\%), oleylamine (OLA, Sigma-Aldrich, 80-90\%), trioctylphosphine (TOP, Sigma-Aldrich, 97\%), n-hexane (VWR, 99\%), ethanol absolute anhydrous (EtOH, Carlo Erba, 99.5\%), methanol ( $\mathrm{MeOH}$, Carlo Erba, 99.8\%), toluene (VWR, 99.3\%), chloroform (Carlo Erba), lithium perchlorate $\left(\mathrm{LiClO}_{4}\right.$, Sigma-Aldrich, 98\%), polyethylene glycol (PEG 6k, $\mathrm{M}_{\mathrm{w}}=6 \cdot 10^{3} \mathrm{~g} \cdot \mathrm{mol}^{-1}$, Fluka).

HgSe CQDs with azobenzene grafting: in a $50 \mathrm{~mL}$ three neck flask, $0.5 \mathrm{~g}$ of $\mathrm{Hg}(\mathrm{OAc})_{2}, 10 \mathrm{~mL}$ of $\mathrm{OA}$ and $25 \mathrm{~mL}$ of OLA were degassed at $85{ }^{\circ} \mathrm{C}$ for $1 \mathrm{~h}$. Under argon atmosphere at $90^{\circ} \mathrm{C}, 1.6 \mathrm{~mL}$ of a $1 \mathrm{M}$ solution of Se in TOP was quickly 
injected into the flask. After $4 \mathrm{~min}$, the reaction was quenched by adding quickly a mix of $1 \mathrm{~mL}$ of $\mathrm{CHCl}_{3}$ and $45 \mathrm{mg}$ of azobenzenes, and cooled down to room temperature using ice bath. The obtained dark solution was then opened to air, precipitated by addition of ethanol and methanol and centrifuged. The supernatant was discarded and the pellet was redispersed in chloroform. The cleaning procedure was repeated two more times. The average nanoparticle diameter in this fraction was $4.3 \pm 0.7 \mathrm{~nm}$, according to the measure by transmission electron microscopy (TEM), see Figure S2. The intraband absorption peak was measured at $3080 \mathrm{~cm}^{-1}$.

HgTe CQDs with azobenzene grafting: $513 \mathrm{mg}$ of $\mathrm{HgCl}_{2}$ was added to $60 \mathrm{~mL}$ of OLA in a $100 \mathrm{~mL}$ round flask. The solution was placed under vacuum and heated to $110^{\circ} \mathrm{C}$ for $1 \mathrm{~h}$. Then, the temperature was decreased to $80^{\circ} \mathrm{C}$ and the solution was placed under Ar atmosphere. $1.9 \mathrm{~mL}$ of TOP:Te (1 M) with $10 \mathrm{~mL}$ of OLA were added to the mercury solution. The solution color immediately turns to dark brown and the reaction was stopped after $3 \mathrm{~min}$. A solution made of $35 \mathrm{mg}$ of azobenzenes and $10 \mathrm{~mL}$ of chloroform was quickly added to quench the reaction. The nanocrystals were then precipitated with ethanol. After centrifugation, the nanocrystals were redispersed in chloroform. The washing step was repeated one more time. The interband peak was measured to be at $4860 \mathrm{~cm}^{-1}$.

\section{Material characterization}

Analytical thin layer chromatography (TLC) was carried out on Merck silica gel60 $F_{254}$. Products were revealed by ultraviolet light ( 254 or $366 \mathrm{~nm}$ ) and stained with dyeing reagents solutions as potassium permanganate solution. Flash chromatography was performed on Combiflash ${ }^{\circledR}$ Companion or with Merck silica gel 60 (230-400 mesh).

${ }^{1} \mathrm{H}$ and ${ }^{13} \mathrm{C}$ NMR spectra were recorded at ambient temperature on Bruker Ascend ${ }^{\mathrm{TM}} 400$ spectrometer operating at $400 \mathrm{MHz}{ }^{1} \mathrm{H} .{ }^{13} \mathrm{C}$ nucleus was observed with ${ }^{1} \mathrm{H}$ decoupling. Solvent residual signals were used as internal standard. Chemical shifts $(\delta)$ in ppm. The peaks patterns are indicated as the following format multiplicity (s: singlet; d: doublet; t: triplet; q: quartet; sept: septuplet; m: multiplet; dd: doublet of doublet; dt: doublet of triplet; dm: doublet of multiplet, etc.). The prefix br. indicates a broadened signal.

UV and visible spectroscopy: Spectra have been acquired using a JASCO V-730 spectrometer.

Infrared spectroscopy: FTIR (Fourier Transform Infrared Spectroscopy) spectra are acquired using a Fischer Nicolet iS50 in ATR configuration. The spectra are averaged over 32 acquisitions and have a $4 \mathrm{~cm}^{-1}$ resolution.

TEM: For TEM imaging, the solution of nanocrystals is drop-cast on a copper TEM grid and dried overnight under vacuum to remove remaining solvent. The TEM images are then obtained using a JEOL 2010 microscope operated at $200 \mathrm{kV}$.

\section{Transport measurements}

Electrodes fabrication: The surface of a $\mathrm{Si} / \mathrm{SiO}_{2}$ wafer ( $400 \mathrm{~nm}$ oxide layer) was cleaned by sonication in acetone. The wafer was rinsed with isopropanol and finally cleaned using an $\mathrm{O}_{2}$ plasma. AZ 5214E resist was spin-coated and baked at $110{ }^{\circ} \mathrm{C}$ for $90 \mathrm{~s}$. The substrate was exposed under UV through a pattern mask for $2 \mathrm{~s}$. The film was further baked at $125^{\circ} \mathrm{C}$ for $2 \mathrm{~min}$ to invert the resist. Then a $40 \mathrm{~s}$ flood exposure was performed. The resist was developed using a bath of AZ 326 for $32 \mathrm{~s}$, before being rinsed in pure water. A $5 \mathrm{~nm}$ chromium layer and $80 \mathrm{~nm}$ gold layer were deposed by using a thermal evaporator. The lift-off was performed by dipping the film in acetone for $1 \mathrm{~h}$. The electrodes were finally rinsed using isopropanol and dried by an air flow. The electrodes were $2.5 \mathrm{~mm}$ long and spaced by $20 \mu \mathrm{m}$. These electrodes were used for DC measurements (IV curves and transistor measurements).

Film preparation: Films were prepared using spin-coating deposition onto electrodes. The solutions were diluted to $20 \mathrm{mg} / \mathrm{mL}$ and spin-coated onto substrates at a speed of $600 \mathrm{rpm}$, with an acceleration of $1000 \mathrm{rpm} / \mathrm{s}$ for $20 \mathrm{~s}$. After drying, the film was dipped in an ethanol solution to remove any remaining free azobenzene ligand. This is a crucial 
step because free azobenzene can percolate and rotate into the film and so their dipole can be orientated in any direction, leading to less efficient surface gating.

Dependency of dark current with dipole: The current of a film of $\mathrm{HgX}(\mathrm{X}=\mathrm{Te}$, Se) nanocrystals grafted with AZB was measured with a Keithley 2634b, with an applied voltage of 0.5 to $1 \mathrm{~V}$. Light (from LED flashlights in the UV at $365 \mathrm{~nm}$ $\left(15 \mathrm{~mW} / \mathrm{cm}^{2}\right)$ and in the blue at $460 \mathrm{~nm}\left(100 \mathrm{~mW} / \mathrm{cm}^{2}\right)$ was shone on the film for $30 \mathrm{~s}$ to change azobenzene configuration and so, the surface dipole. Dark current after illumination was measured once the current value is stabilized.

Transistor measurements using electrolyte gating: For electrolyte gating, we first mix, in a glove box, $0.5 \mathrm{~g}$ of $\mathrm{LiClO}_{4}$, with $2.3 \mathrm{~g}$ of PEG $\left(\mathrm{MW}_{\mathrm{w}}=6 \mathrm{~kg} \cdot \mathrm{mol}^{-1}\right)$. The vial is heated at $170^{\circ} \mathrm{C}$ on a hot plate for $2 \mathrm{~h}$ until the solution gets clear. To use the electrolyte, the solution is warmed around $100{ }^{\circ} \mathrm{C}$ and brushed on the top of the HgTe film. The sample is then connected to a Keithley $2634 \mathrm{~b}$ which sets the drain bias $\left(\mathrm{V}_{\mathrm{DS}}=200-500 \mathrm{mV}\right)$, controls the gate bias $\left(\mathrm{V}_{\mathrm{GS}}\right)$ with a step of $1 \mathrm{mV}$ and measures the associated currents $\mathrm{IDS}_{\mathrm{DS}}$ and $\mathrm{IGS}_{\mathrm{GS}}$
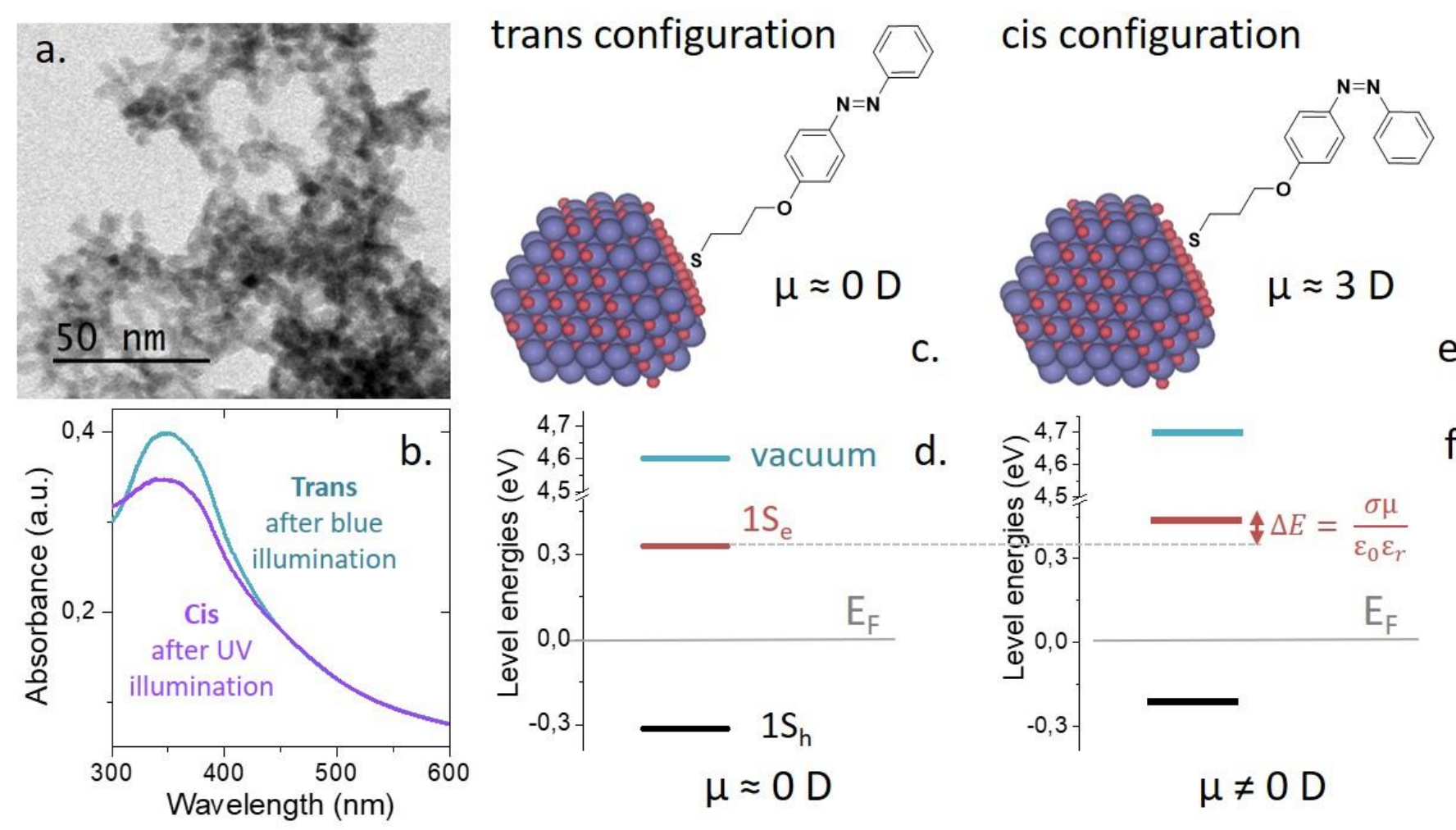

e.

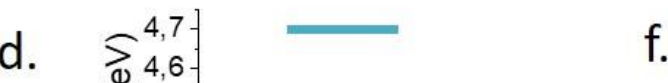

Figure 2 a. TEM picture of HgTe NCs with AZB grafted at the surface. b. Absorption spectra of AZB grafted HgTe NCS after UV $(\lambda=365 \mathrm{~nm})$ and blue illumination $(\lambda=460 \mathrm{~nm})$. c. Scheme of HgTe NCs grafted with AZB in trans-configuration. d. Electronic spectrum of the HgTe NCs with pristine surface. e. Scheme of HgTe NCs with AZB in cis-configuration. $f$. Electronic spectrum of the HgTe NCs with cis AZB grafted.

\section{DISCUSSION}

As the band shift induced by surface dipole is not depending on the material band gap, we choose to work with narrow band gap materials which will present a larger band gap renormalization upon the change of AZB configuration. In other words, the effect of band shift in those nanocrystals should be a significant fraction of their bandgap energy. We use NCs of HgTe and HgSe according to established procedures given respectively in ref 35 and 19. These two materials have attracted a significant interest for the design of IR detectors ${ }^{36-40}$ with tunable absorption from short-wave infrared and up to $\mathrm{THz}^{41}$ In such arrays of $\mathrm{NCs}$, infrared detection is possible but the performance (dark current, spectral and time response) dramatically depends on the doping magnitude. It is thus of utmost interest to have an external knob to tune the carrier density.

Both materials have a zinc blende structure, according to their diffraction pattern (figure S1). HgTe presents a tetrapodic shape, see Figure 2a with 8-nm-long arm, while HgSe is more spherical (Figure S2) with a size around 4.7 $\mathrm{nm}$. Using a combination of photoemission ${ }^{42}$ and infrared spectroscopy (see figure S3 for procedure details) it is 
possible to determine the electronic spectrum of each material in absolute energy scale (i.e. vs vacuum). These two materials have been chosen because they present $\mathrm{p}(\mathrm{HgTe})$ and $\mathrm{n}(\mathrm{HgSe})$ nature. $\mathrm{HgTe}$ is quasi intrinsic, with a Fermi level in the lower part of the band gap, leading to small p-type nature ${ }^{43}$, see Figure $2 \mathrm{~d}$, while $\mathrm{HgSe}$ is degenerately $\mathrm{n}$ doped as discussed later in the text. ${ }^{18,19}$

The goal of this paper is to demonstrate that the surface dipole resulting from the AZB can be tuned using light. To graft the AZBs on the mercury chalcogenides NCs, we take advantage of the strong affinity of surface mercury cations for sulfur. The AZB is first functionalized with a thiol, according to the procedure given in Figure 1 and Figure S4-6. The thiol-functionalized AZB is then used to quench the nanocrystal growth. The confirmation of the grafting is obtained by UV-visible (Figure $2 \mathrm{~b}$ and $\mathrm{S6}$ ) and infrared spectroscopies (Figure S7). In particular, we observe a strong blueshift of the UV absorption spectrum of the AZB grafted NCs after UV illumination $\left(\lambda=365 \mathrm{~nm} ; \mathrm{P}<<\mathrm{W} . \mathrm{cm}^{-2}\right)$ which can be associated to the cis conformation of the AZB. After illumination in the visible $\left(\lambda=460 \mathrm{~nm} ; \mathrm{P}<<W . \mathrm{cm}^{-2}\right)$, this band redshifts, which is consistent with the switching to the trans conformation of the AZB. We expect that the lighttriggered switching of the ligand conformation increases the surface dipole which will result in a band shifting. As a result, the relative position of these bands with respect to the Fermi level is tuned leading to a change in the nanocrystal carrier density.

We start our study with p-type mercury telluride nanocrystals. Their transport properties are first probed in an electrolyte-gated field effect transistor (FET) configuration. ${ }^{44} \mathrm{~A}$ scheme of the device can be seen in Figure 3a. Ion gel gating approach has been quite successful to achieve gating on mercury chalcogenides nanocrystals. ${ }^{45}$ In addition of air operability, this approach leads to large gate capacitance which can tune the carrier density up to several carriers per nanocrystal. ${ }^{46} \mathrm{HgTe}$ nanocrystals present an ambipolar behavior, with a higher mobility for the holes than for electrons (Figure $3 \mathrm{~b}$ ), which is expected with the electronic spectrum proposed in Figure $2 \mathrm{~d}$. The mobility of the carrier is estimated to be 50 times smaller than for conventionally used short dithiol (ethanedithiol), see figure S8. Another striking difference between EDT and AZB capping is the fact than AZB present stronger hole conduction compared to electron conduction, while the opposite behavior is observed for EDT. This suggests than AZB favor the p-doping of the HgTe. ${ }^{47}$

We then use transport measurement as a probe of the change of carrier density with AZB conformation. We follow charge transport as a function of time after pulses of UV and visible light to switch the conformation of the AZB, see Figure 3c. During the illumination, we observe a large increase of the current. This current modulation is due to photocharge generation within the HgTe semiconductor. Then as the sample returns to dark condition, we first see a transient current. The latter is not due to transient photocurrent since the response of $\mathrm{HgTe}$ is fast $\mathrm{fr}^{48}(>\mathrm{MHz}$ and see figure S9). This regime actually reflects that AZB conformation thermally comes back to trans. Finally, the dark current stabilizes at a value higher than before UV exposure. This modulation of the current is the signature of the change of AZB dipole on the NC surface. After a pulse of visible light, the current in the dark recovers its initial value. The AZB change of conformation is fairly reversible over the first cycles, as shown in Figure $3 \mathrm{~d}$ and S10. For sake of comparison, we have also checked that the sample without AZB grafted, does not lead to a persistent modulation of the dark current after the illumination, see Figure 59. 

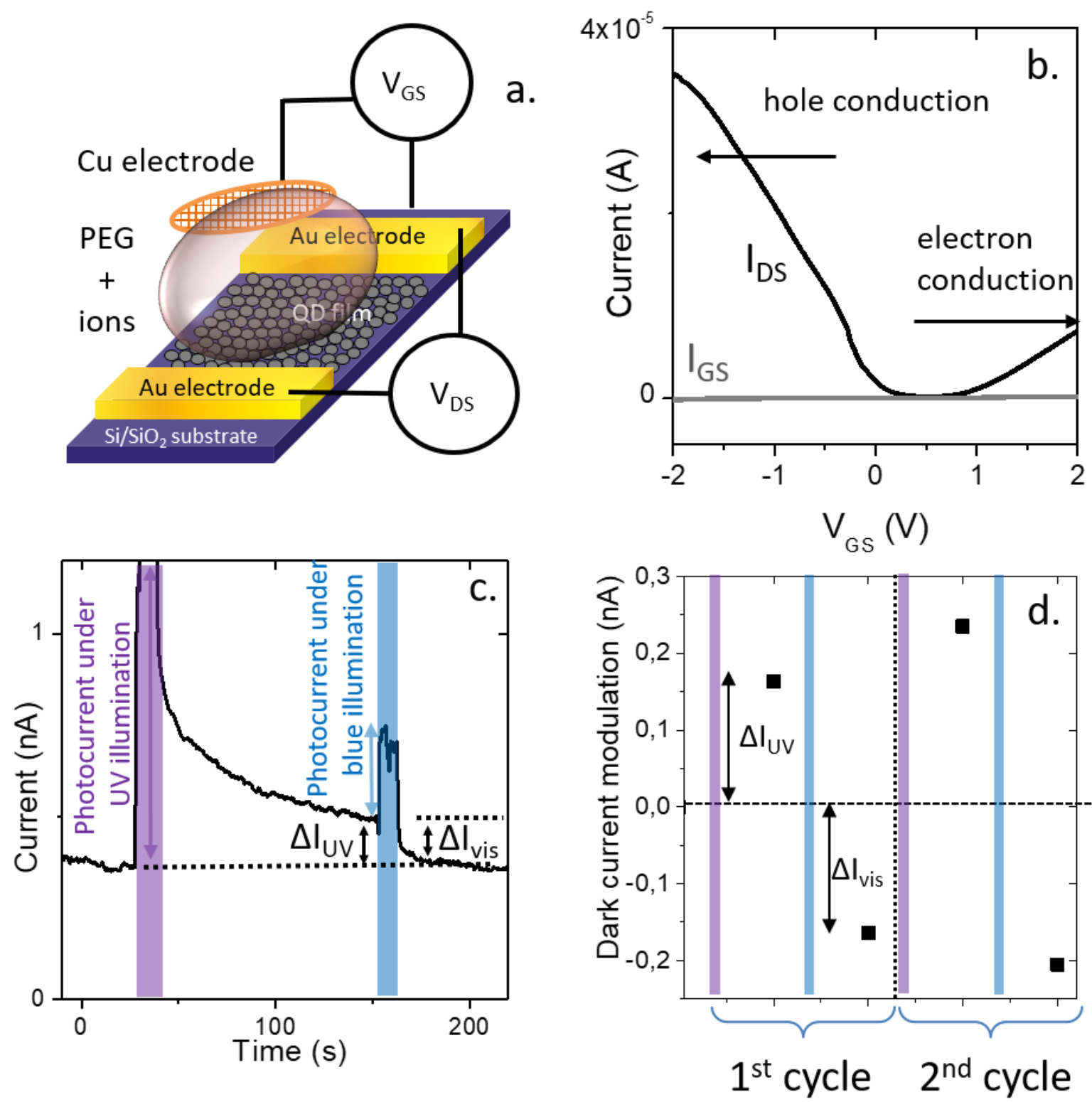

Figure 3 a. Scheme of an electrolyte gated field effect transistor (FET) which channel is made of a thin film of HgTe. $b$. Transfer curve (drain-source current as a function of gate bias) for the FET based on AZB functionalized HgTe channel c. Drain source current of an HgTe capped with azobenzene film as a function of time. UV ( $\lambda=365 \mathrm{~nm})$ or blue light $(\lambda=460 \mathrm{~nm})$ is shone on the film during the intervals highlighted with purple and blue rectangles respectively. $\Delta l u v$ is the change of current as the $A Z B$ switches from trans to cis, while $\triangle I_{v i s}$ is the change of current as the $A Z B$ switches from cis to trans $d$. Current modulation (as shown on Figure $3 c$ ) induced by UV and visible pulse of light as a function of pulse event.

Assuming a value of $\mu=2.5 \times 10^{-5} \mathrm{~cm}^{2} \mathrm{~V}^{-1} \mathrm{~s}^{-1}$ (see Supplementary information) for the carrier mobility, we can estimate the change of carrier density $\Delta n$ within the nanocrystal $\Delta n=\frac{\Delta j}{e \mu F^{\prime}}$, with $\Delta j$ the change of current density, $e$ the elementary charge and $F$ the electric field. The initial hole density in this p-type material is $1.8 \times 10^{-4}$ thermally activated holes per nanocrystal and it increases after the light activation of the surface dipole to $2.6 \times 10^{-4}$. Because the hole carrier density is far below 1 per nanocrystal, we can assume that only the $1 S_{h}$ state is filled. We can thus estimate the relative position of this level with respect to the Fermi level $\left(\mathrm{E}_{\mathrm{F}}\right) p=2 f_{F D}\left(1 S_{h}-E_{F}\right)$ with $\mathrm{f}_{\mathrm{FD}}$ the Fermi Dirac distribution. Thus, the observed change of current is the result of a $7 \mathrm{meV}$ shift of the valence band toward the Fermi level. Knowing this shift, we can determine the surface density of AZB on the HgTe. We found typically $\approx 3$ AZBs per nanocrystal. This rather low value can be explained by the tetrapodic shape of the HgTe nanoparticle and the bulkiness of the AZB, which conformation change also limits the grafting density. 

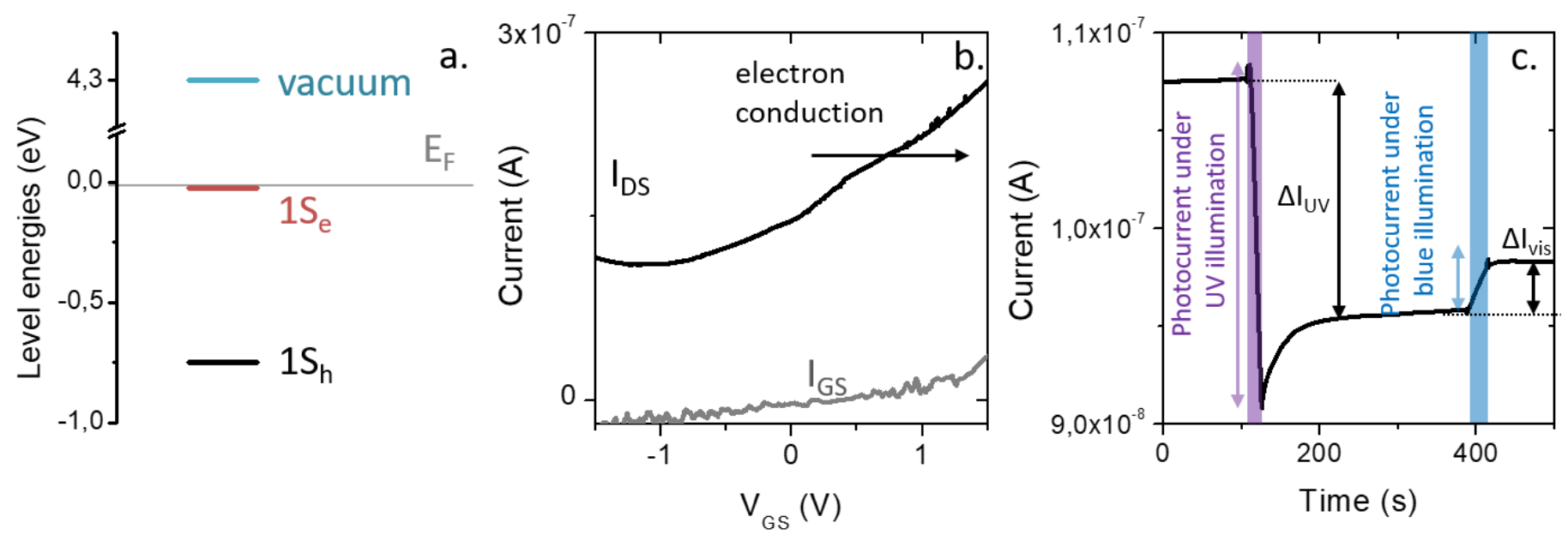

Figure 4 a. Electronic spectrum of the HgSe with pristine surface. $b$. Transfer curve (current as a function of gate bias) for the FET based on HgSe channel. c. Drain source current of an HgTe capped with azobenzene film as a function of time. UV $(\lambda=365 \mathrm{~nm})$ or blue light $(\lambda=460 \mathrm{~nm})$ is shone on the film during the intervals highlighted with purple and blue bars respectively.

To further enhance the grafting density of AZB on the nanocrystals surface, we then switch to HgSe NCs which spherical shape is more favorable. ${ }^{49,50}$ The other striking interest to use HgSe is to have a doping level very different from HgTe. $\mathrm{HgSe}$ is indeed an example of degenerately $\mathrm{n}$-doped material. The size of the NC has been chosen to have a full filled $1 \mathrm{~S}_{\mathrm{e}}$ state, see the electronic spectrum in Figure $4 \mathrm{a}$ and the transistor transfer curve in Figure $4 \mathrm{~b}$ which confirm that the material only presents a n-type character. In p-doped material (i.e. HgTe), the dipole-related shift of the band was increasing the hole density. Because the shift direction is the same for HgSe, it should induce a drop in the electron carrier density.

It is worth noting that the degenerate doping makes that, under the low excitation power used here, the photocurrent signal is negligible compared to the dark current. As a result, the effect of the light on HgSe sample is mostly the result of the dark current modulation. This contrasts with HgTe where photocurrent modulation was large due to a lower carrier density. As we follow the current in the sample as a function of time, we indeed observe a significant decrease of the current in the steady state after UV illumination (Figure 4c). After illumination by blue visible light (return to the trans configuration), the current increases again, even if the reversibility is not as good. With the same procedure as before, we estimate the change of carrier density from 1.94 electrons per dot down to 1.72 electrons in the AZB cis configuration. This corresponds to a $40 \mathrm{meV}$ shift for the relative energy of the $1 \mathrm{~S}_{\mathrm{e}}$ state with respect to the Fermi level and to approximately 8 AZBs per HgSe NC.

\section{CONCLUSION}

In conclusion, we have demonstrated the remote control of the carrier density thanks to mechanized nanocrystals. The emergence of the functionality at the macroscopic level comes from the grafting of thiol functionalized Azobenzene at the surface of $\mathrm{HgTe} / \mathrm{HgSe}$ nanoparticles. This molecular machine has been selected for its dipole moment shift resulting from the reversible conformation switches upon irradiation. The AZB conformation switches towards the cis conformation and leads to an increase of the hole density for $p$-type materials and a reduction of the electron density for $n$-type materials. This method allows the tuning of carrier density using low irradiance $(<100$ $\mathrm{mW} . \mathrm{cm}^{-2}$ ) which does not damage the sample through heating. Further work will have to focus on the increase of the AZB surface density of the NC surface and on boosting reversibility. 


\section{ACKNOWLEDEGMENTS}

EL thanks the support ERC starting grant blackQD (grant $n^{\circ}$ 756225), while SI thanks the support of ERC starting grant Ne2Dem. We acknowledge the use of clean-room facilities from the "Centrale de Proximité Paris-Centre". This work has been supported by the Region lle-de-France in the framework of DIM Nano-K (grant dopQD). This work was supported by French state funds managed by the ANR within the Investissements d'Avenir programme under reference ANR-11-IDEX-0004-02, and more specifically within the framework of the Cluster of Excellence MATISSE and also by the grant IPER-Nano, Copin, Graskop, Frontal. JQ thanks Chinese Scholar council for PhD grant.

\section{REFERENCES}

(1) Konstantatos, G.; Sargent, E. H. Colloidal Quantum Dot Optoelectronics and Photovoltaics; Cambridge University Press, 2013.

(2) Talapin, Dmitri V.; Lee, Jong Soo; Kovalenko, Maksym V.; Shevchenko, Elena V. Prospects of Colloidal Nanocrystals for Electronic and Optoelectronic Applications. Chem Rev 2010, 110, 389-458. https://doi.org/10.1021/cr900137k.

(3) Wood, V.; Bulović, V. Colloidal Quantum Dot Light-Emitting Devices. Nano Rev. 2010, 1, 5202. https://doi.org/10.3402/nano.v1i0.5202.

(4) Semonin, O. E.; Luther, J. M.; Choi, S.; Chen, H.-Y.; Gao, J.; Nozik, A. J.; Beard, M. C. Peak External Photocurrent Quantum Efficiency Exceeding 100\% via MEG in a Quantum Dot Solar Cell. Science 2011, 334, 1530-1533. https://doi.org/10.1126/science.1209845.

(5) McDonald, S. A.; Konstantatos, G.; Zhang, S.; Cyr, P. W.; Klem, E. J. D.; Levina, L.; Sargent, E. H. SolutionProcessed PbS Quantum Dot Infrared Photodetectors and Photovoltaics. Nat. Mater. 2005, 4, 138-142. https://doi.org/10.1038/nmat1299.

(6) Keuleyan, S.; Lhuillier, E.; Brajuskovic, V.; Guyot-Sionnest, P. Mid-Infrared HgTe Colloidal Quantum Dot Photodetectors. Nat. Photonics 2011, 5 (8), 489-493. https://doi.org/10.1038/nphoton.2011.142.

(7) Chuang, C.-H. M.; Brown, P. R.; Bulović, V.; Bawendi, M. G. Improved Performance and Stability in Quantum Dot Solar Cells through Band Alignment Engineering. Nat. Mater. 2014, 13, 796-801. https://doi.org/10.1038/nmat3984.

(8) Christodoulou, S.; Ramiro, I.; Othonos, A.; Figueroba, A.; Dalmases, M.; Özdemir, O.; Pradhan, S.; Itskos, G.; Konstantatos, G. Single-Exciton Gain and Stimulated Emission Across the Infrared Optical Telecom Band from Robust Heavily-Doped PbS Colloidal Quantum Dots. arXiv:1908.03796 2019.

(9) Jagtap, A.; Livache, C.; Martinez, B.; Qu, J.; Chu, A.; Gréboval, C.; Goubet, N.; Lhuillier, E. Emergence of Intraband Transitions in Colloidal Nanocrystals. Opt. Mater. Express 2018, 8, 1174-1183. https://doi.org/10.1364/OME.8.001174.

(10) Wang, C.; Shim, M.; Guyot-Sionnest, P. Electrochromic Nanocrystal Quantum Dots. Science 2001, 291, 23902392. https://doi.org/10.1126/science.291.5512.2390.

(11) Schimpf, A. M.; Knowles, K. E.; Carroll, G. M.; Gamelin, D. R. Electronic Doping and Redox-Potential Tuning in Colloidal Semiconductor Nanocrystals. Acc. Chem. Res. 2015, 48, 1929-1937. https://doi.org/10.1021/acs.accounts.5b00181.

(12) Erwin, S. C.; Zu, L.; Haftel, M. I.; Efros, A. L.; Kennedy, T. A.; Norris, D. J. Doping Semiconductor Nanocrystals. Nature 2005, 436, 91-94. https://doi.org/10.1038/nature03832.

(13) Rinehart, J. D.; Schimpf, A. M.; Weaver, A. L.; Cohn, A. W.; Gamelin, D. R. Photochemical Electronic Doping of Colloidal CdSe Nanocrystals. J. Am. Chem. Soc. 2013, 135, 18782-18785. https://doi.org/10.1021/ja410825c.

(14) Nag, A.; Sapra, S.; Nagamani, C.; Sharma, A.; Pradhan, N.; Bhat, S. V.; Sarma, D. D. A Study of Mn2+ Doping in CdS Nanocrystals. Chem. Mater. 2007, 19, 3252-3259. https://doi.org/10.1021/cm0702767.

(15) Knowles, K. E.; Hartstein, K. H.; Kilburn, T. B.; Marchioro, A.; Nelson, H. D.; Whitham, P. J.; Gamelin, D. R. Luminescent Colloidal Semiconductor Nanocrystals Containing Copper: Synthesis, Photophysics, and Applications. Chem. Rev. 2016, 116, 10820-10851. https://doi.org/10.1021/acs.chemrev.6b00048.

(16) Sahu, A.; Kang, M. S.; Kompch, A.; Notthoff, C.; Wills, A. W.; Deng, D.; Winterer, M.; Frisbie, C. D.; Norris, D. J. Electronic Impurity Doping in CdSe Nanocrystals. Nano Lett. 2012, 12, 2587-2594. https://doi.org/10.1021/nl300880g.

(17) Luther, J. M.; Jain, P. K.; Ewers, T.; Alivisatos, A. P. Localized Surface Plasmon Resonances Arising from Free Carriers in Doped Quantum Dots. Nat. Mater. 2011, 10, 361-366. https://doi.org/10.1038/nmat3004. 
(18) Deng, Z.; Jeong, K. S.; Guyot-Sionnest, P. Colloidal Quantum Dots Intraband Photodetectors. ACS Nano 2014, 8, 11707-11714. https://doi.org/10.1021/nn505092a.

(19) Lhuillier, E.; Scarafagio, M.; Hease, P.; Nadal, B.; Aubin, H.; Xu, X. Z.; Lequeux, N.; Patriarche, G.; Ithurria, S.; Dubertret, B. Infrared Photodetection Based on Colloidal Quantum-Dot Films with High Mobility and Optical Absorption up to THz. Nano Lett. 2016, 16, 1282-1286. https://doi.org/10.1021/acs.nanolett.5b04616.

(20) Mahler, B.; Guillemot, L.; Bossard-Giannesini, L.; Ithurria, S.; Pierucci, D.; Ouerghi, A.; Patriarche, G.; Benbalagh, R.; Lacaze, E.; Rochet, F.; et al. Metallic Functionalization of CdSe 2D Nanoplatelets and Its Impact on Electronic Transport. J. Phys. Chem. C 2016, 120, 12351-12361. https://doi.org/10.1021/acs.jpcc.6b02101.

(21) Lee, J.-S.; Shevchenko, E. V.; Talapin, D. V. Au-PbS Core-Shell Nanocrystals: Plasmonic Absorption Enhancement and Electrical Doping via Intra-Particle Charge Transfer. J. Am. Chem. Soc. 2008, 130, 9673-9675. https://doi.org/10.1021/ja802890f.

(22) Koh, W.; Koposov, A. Y.; Stewart, J. T.; Pal, B. N.; Robel, I.; Pietryga, J. M.; Klimov, V. I. Heavily Doped $n$-Type PbSe and PbS Nanocrystals Using Ground-State Charge Transfer from Cobaltocene. Sci. Rep. 2013, 3, 2004. https://doi.org/10.1038/srep02004.

(23) Martinez, B.; Livache, C.; Meriggio, E.; Xu, X. Z.; Cruguel, H.; Lacaze, E.; Proust, A.; Ithurria, S.; Silly, M. G.; Cabailh, G.; et al. Polyoxometalate as Control Agent for the Doping in HgSe Self-Doped Nanocrystals. J. Phys. Chem. C 2018, 122, 26680-26685. https://doi.org/10.1021/acs.jpcc.8b07190.

(24) Miller, E. M.; Kroupa, D. M.; Zhang, J.; Schulz, P.; Marshall, A. R.; Kahn, A.; Lany, S.; Luther, J. M.; Beard, M. C.; Perkins, C. L.; et al. Revisiting the Valence and Conduction Band Size Dependence of PbS Quantum Dot Thin Films. ACS Nano 2016, 10, 3302-3311. https://doi.org/10.1021/acsnano.5b06833.

(25) Kroupa, D. M.; Vörös, M.; Brawand, N. P.; McNichols, B. W.; Miller, E. M.; Gu, J.; Nozik, A. J.; Sellinger, A.; Galli, G.; Beard, M. C. Tuning Colloidal Quantum Dot Band Edge Positions through Solution-Phase Surface Chemistry Modification. Nat. Commun. 2017, 8, 15257. https://doi.org/10.1038/ncomms15257.

(26) Robin, A.; Livache, C.; Ithurria, S.; Lacaze, E.; Dubertret, B.; Lhuillier, E. Surface Control of Doping in Self-Doped Nanocrystals. ACS Appl. Mater. Interfaces 2016, 8, 27122-27128. https://doi.org/10.1021/acsami.6b09530.

Brown, P. R.; Kim, D.; Lunt, R. R.; Zhao, N.; Bawendi, M. G.; Grossman, J. C.; Bulović, V. Energy Level Modification in Lead Sulfide Quantum Dot Thin Films through Ligand Exchange. ACS Nano 2014, 8, 5863-5872. https://doi.org/10.1021/nn500897c.

(28) Martinez, B.; Livache, C.; Notemgnou Mouafo, L. D.; Goubet, N.; Keuleyan, S.; Cruguel, H.; Ithurria, S.; Aubin, H.; Ouerghi, A.; Doudin, B.; et al. HgSe Self-Doped Nanocrystals as a Platform to Investigate the Effects of Vanishing Confinement. ACS Appl. Mater. Interfaces 2017, 9, 36173-36180. https://doi.org/10.1021/acsami.7b10665.

(29) Gréboval, C.; Noumbe, U.; Goubet, N.; Livache, C.; Ramade, J.; Qu, J.; Chu, A.; Martinez, B.; Prado, Y.; Ithurria, S.; et al. Field-Effect Transistor and Photo-Transistor of Narrow-Band-Gap Nanocrystal Arrays Using lonic Glasses. Nano Lett. 2019, 19, 3981-3986. https://doi.org/10.1021/acs.nanolett.9b01305.

(30) Talapin, D. V.; Murray, C. B. PbSe Nanocrystal Solids for N- and p-Channel Thin Film Field-Effect Transistors. Science 2005, 310, 86-89. https://doi.org/10.1126/science.1116703.

(31) Peng, K.; Tomatsu, I.; Kros, A. Light Controlled Protein Release from a Supramolecular Hydrogel. Chem. Commun. 2010, 46, 4094-4096. https://doi.org/10.1039/C002565H.

(32) Zhao, H.; Sen, S.; Udayabhaskararao, T.; Sawczyk, M.; Kučanda, K.; Manna, D.; Kundu, P. K.; Lee, J.-W.; Král, P.; Klajn, R. Reversible Trapping and Reaction Acceleration within Dynamically Self-Assembling Nanoflasks. Nat. Nanotechnol. 2016, 11, 82-88. https://doi.org/10.1038/nnano.2015.256.

(33) Credi, A. Artificial Molecular Motors Powered by Light. Aust. J. Chem. 2006, 59 (3), 157-169. https://doi.org/10.1071/CH06025.

(34) Hartley, G. S.; Fèvre, R. J. W. L. The Dipole Moments of Cis- and Trans-Azobenzenes and of Some Related Compounds. J. Chem. Soc. Resumed 1939, 531-535. https://doi.org/10.1039/JR9390000531.

(35) Keuleyan, S.; Lhuillier, E.; Guyot-Sionnest, P. Synthesis of Colloidal HgTe Quantum Dots for Narrow Mid-IR Emission and Detection. J. Am. Chem. Soc. 2011, 133, 16422-16424. https://doi.org/10.1021/ja2079509.

(36) Lhuillier, E.; Guyot-Sionnest, P. Recent Progresses in Mid Infrared Nanocrystal Based Optoelectronics. IEEE J. Sel. Top. Quantum Electron. 2017, 23, 6000208. https://doi.org/10.1109/JSTQE.2017.2690838.

(37) Cryer, M. E.; Halpert, J. E. $300 \mathrm{Nm}$ Spectral Resolution in the Mid-Infrared with Robust, High Responsivity Flexible Colloidal Quantum Dot Devices at Room Temperature. ACS Photonics 2018, 5, 3009-3015. https://doi.org/10.1021/acsphotonics.8b00738.

(38) Chen, M.; Lu, H.; Abdelazim, N. M.; Zhu, Y.; Wang, Z.; Ren, W.; Kershaw, S. V.; Rogach, A. L.; Zhao, N. Mercury Telluride Quantum Dot Based Phototransistor Enabling High-Sensitivity Room-Temperature Photodetection at 2000 Nm. ACS Nano 2017, 11, 5614-5622. https://doi.org/10.1021/acsnano.7b00972. 
(39) Tang, X.; Ackerman, M. M.; Chen, M.; Guyot-Sionnest, P. Dual-Band Infrared Imaging Using Stacked Colloidal Quantum Dot Photodiodes. Nat. Photonics 2019, 13, 277-282. https://doi.org/10.1038/s41566-019-0362-1.

(40) Livache, C.; Martinez, B.; Goubet, N.; Gréboval, C.; Qu, J.; Chu, A.; Royer, S.; Ithurria, S.; Silly, M. G.; Dubertret, B.; et al. A Colloidal Quantum Dot Infrared Photodetector and Its Use for Intraband Detection. Nat. Commun. 2019, 10, 2125. https://doi.org/10.1038/s41467-019-10170-8.

(41) Goubet, N.; Jagtap, A.; Livache, C.; Martinez, B.; Portalès, H.; Xu, X. Z.; Lobo, R. P. S. M.; Dubertret, B.; Lhuillier, E. Terahertz HgTe Nanocrystals: Beyond Confinement. J. Am. Chem. Soc. 2018, 140, 5033-5036. https://doi.org/10.1021/jacs.8b02039.

(42) Sarma, D. D.; Santra, P. K.; Mukherjee, S.; Nag, A. X-Ray Photoelectron Spectroscopy: A Unique Tool To Determine the Internal Heterostructure of Nanoparticles. Chem. Mater. 2013, 25, 1222-1232. https://doi.org/10.1021/cm303567d.

(43) Chu, A.; Martinez, B.; Ferré, S.; Noguier, V.; Gréboval, C.; Livache, C.; Qu, J.; Prado, Y.; Casaretto, N.; Goubet, N.; et al. HgTe Nanocrystals for SWIR Detection and Their Integration up to the Focal Plane Array. ACS Appl. Mater. Interfaces 2019, 11, 33116-33123. https://doi.org/10.1021/acsami.9b09954.

(44) Martinez, B.; Livache, C.; Goubet, N.; Jagtap, A.; Cruguel, H.; Ouerghi, A.; Lacaze, E.; Silly, M. G.; Lhuillier, E. Probing Charge Carrier Dynamics to Unveil the Role of Surface Ligands in HgTe Narrow Band Gap Nanocrystals. J. Phys. Chem. C 2018, 122, 859-865. https://doi.org/10.1021/acs.jpcc.7b09972.

(45) Izquierdo, E.; Robin, A.; Keuleyan, S.; Lequeux, N.; Lhuillier, E.; Ithurria, S. Strongly Confined HgTe 2D Nanoplatelets as Narrow Near-Infrared Emitters. J. Am. Chem. Soc. 2016, 138, 10496-10501. https://doi.org/10.1021/jacs.6b04429.

(46) Lhuillier, E.; Scarafagio, M.; Hease, P.; Nadal, B.; Aubin, H.; Xu, X. Z.; Lequeux, N.; Patriarche, G.; Ithurria, S.; Dubertret, B. Infrared Photodetection Based on Colloidal Quantum-Dot Films with High Mobility and Optical Absorption up to THz. Nano Lett. 2016, 16, 1282-1286. https://doi.org/10.1021/acs.nanolett.5b04616.

(47) Martinez, B.; Livache, C.; Goubet, N.; Jagtap, A.; Cruguel, H.; Ouerghi, A.; Lacaze, E.; Silly, M. G.; Lhuillier, E. Probing Charge Carrier Dynamics to Unveil the Role of Surface Ligands in HgTe Narrow Band Gap Nanocrystals. J. Phys. Chem. C 2018, 122, 859-865. https://doi.org/10.1021/acs.jpcc.7b09972.

(48) Livache, C.; Goubet, N.; Martinez, B.; Jagtap, A.; Qu, J.; Ithurria, S.; Silly, M. G.; Dubertret, B.; Lhuillier, E. Band Edge Dynamics and Multiexciton Generation in Narrow Band Gap HgTe Nanocrystals. ACS Appl. Mater. Interfaces 2018, 10, 11880-11887. https://doi.org/10.1021/acsami.8b00153.

(49) Grigel, V.; Sagar, L. K.; De Nolf, K.; Zhao, Q.; Vantomme, A.; De Roo, J.; Infante, I.; Hens, Z. The Surface Chemistry of Colloidal HgSe Nanocrystals, toward Stoichiometric Quantum Dots by Design. Chem. Mater. 2018, 30, 76377647. https://doi.org/10.1021/acs.chemmater.8b02908.

(50) Nag, A.; Zhang, H.; Janke, E.; Talapin, D. V. Inorganic Surface Ligands for Colloidal Nanomaterials. Z. Für Phys. Chem. 2014, 229, 85-107. https://doi.org/10.1515/zpch-2014-0604. 
TOC graphic

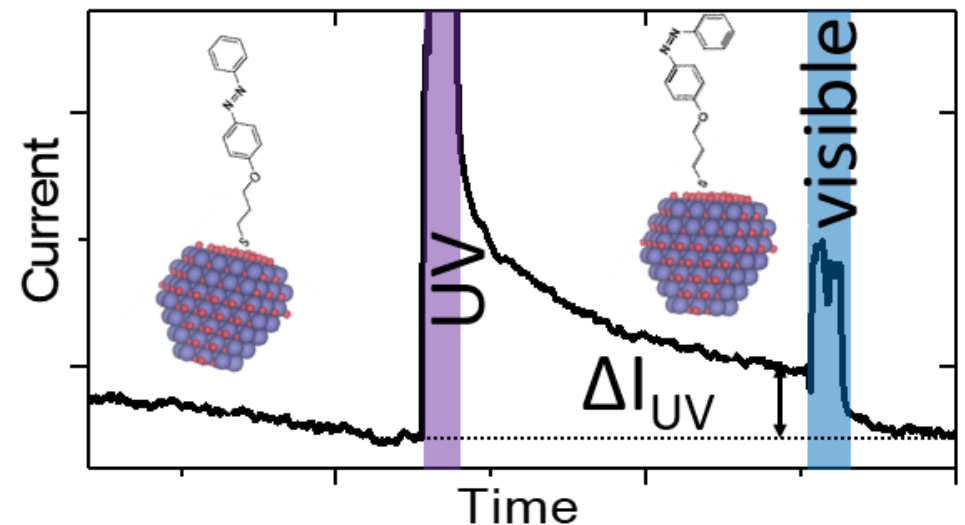

